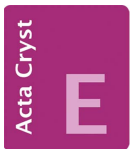

CRYSTALLOGRAPHIC COMMUNICATIONS

ISSN 2056-9890

\section{Synthesis and crystal structure of a new coordination polymer based on lanthanum and 1,4-phenylenediacetate ligands}

\author{
Magatte Camara, ${ }^{\mathrm{a} *}$ Insa Badiane, ${ }^{\mathrm{a}}$ Mamoudou Diallo, ${ }^{\mathrm{a}}$ Carole Daiguebonne ${ }^{\mathrm{b}}$ and \\ Olivier Guilloub
}

Received 6 February 2019

Accepted 15 February 2019

Edited by A. Van der Lee, Université de Montpellier II, France

Keywords: lanthanum ion; coordination polymer; crystal structure.

CCDC reference: 1875083

Supporting information: this article has supporting information at journals.iucr.org/e

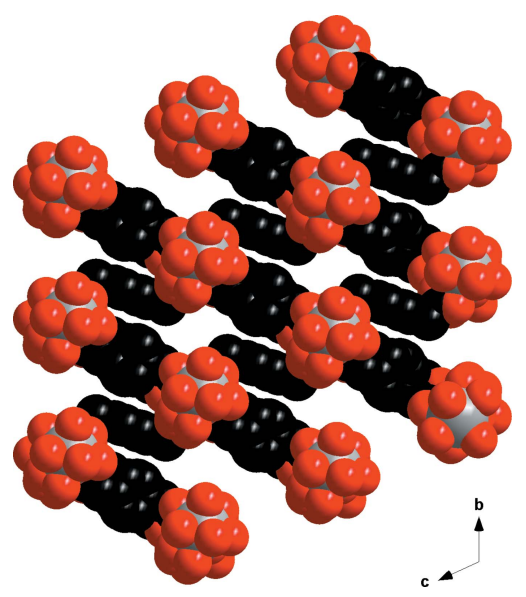

\section{Chemical context}

In recent years, one of the most important fields of research in coordination chemistry and crystal engineering has been the design of metal-organic frameworks (MOFs), because of their intriguing network topologies and possible applications in gas storage (Eddaoudi et al., 2002; Reneike et al., 1999; Luo et al., 2011a,b; Kustaryono et al., 2010), catalysis (Lee et al., 2009), separation (Hamon et al., 2009), luminescence (Cui et al., 2012; Daiguebonne et al., 2008; Binnemans, 2009;) and molecular magnetism (Calvez et al., 2008; Sessoli et al., 2009). Our group has been involved in this field for more than a decade (Freslon et al., 2014; Fan et al., 2014; Luo et al., 2011a,b; Badiane et al., $2017 a, b)$. The search for new ligands that can lead to new structural networks and/or new physical properties is a continuous concern (Qiu et al., 2007; Fan et al., 2015).

For the synthesis of MOFs, usually two complementary molecular precursors, a cation with vacant coordination sites and a bridging anion, are used to form the coordination polymer. This procedure offers the prospect of rationally designing extended solids with interesting properties. Most of the organic ligands used in MOF chemistry are rigid aromatic carboxylates (Luo et al., 2007; Huang et al., 2009). Compared to the rigid ligands, using flexible ligands such as 1,2- (Xin et al., 2011), 1,3- (Wang et al., 2012) or 1,4-phenylenediacetate (Fabelo et al., 2009a,b) to construct coordination polymers seems to be more difficult, and developing synthetic methodologies is still a challenge. However, flexibility of the ligand can promote structural and functional diversity.

Numerous coordination polymers have been reported so far that involve $d$-block metal ions such as $\mathrm{Cu}^{\mathrm{II}}$ (Singh \& Barua, 2009; Fabelo et al., 2009a,b; Chen et al., 2010a,b,c), Zn ${ }^{\mathrm{II}}$ (Singh 
<smiles>COC(Cc1ccc(CC(OC)OC)cc1)OC</smiles>

Mode I

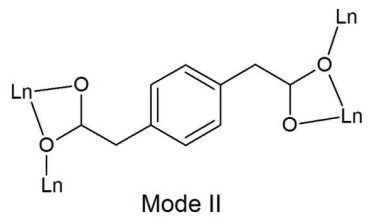<smiles>CCCCOC(Cc1ccc(CC2O[Ga]O2)cc1)O[Ga]</smiles>

Mode III

Figure 1

Bonding modes in lanthanide-containing coordination polymers with 1,4phenylenediacetate ligands $\left(p-\mathrm{pda}^{2-}\right)$ reported in the literature to date.

\& Barua, 2009), $\mathrm{Cd}^{\mathrm{II}}$ (Chen et al., 2010a,b,c; Singh \& Barua, 2009; Li et al., 2009), $\mathrm{Mn}^{\mathrm{II}}$ (Singh \& Barua, 2009; Chen et al., $2010 a, b, c, \mathrm{Co}^{\mathrm{II}}$ (Fabelo et al., 2009a,b; Chen et al., 2010a,b,c; Uebler \& LaDuca, 2012; Li et al., 2009) and $\mathrm{Ni}^{\mathrm{II}}$ (Chen et al., 2010a,b,c; Uebler \& LaDuca, 2012; Li et al., 2009). Lanthanide(III) ions have higher and variable coordination numbers (generally between 7 and 12) and incorporate in addition, apart from the main ligands, ancillary ligands such as water molecules into the lanthanide coordination sphere. A large number of studies have been reported on lanthanide coordination polymers based on 1,4-phenylenediacetic acid (Singh \& Barua, 2009; Fabelo et al., 2009a,b; Chen et al., 2010a,b,c; Uebler \& LaDuca, 2012; Li et al., 2009; Rusinek et al., 2013) as well as on other isomers of this acid such as 1,2- (Badiane et al., 2017a,b; Xin et al., 2011) and 1,3-phenylenediacetic acid (Wang et al., 2012), and most of them tend to make porous materials through solvothermal synthesis.

Isomers of phenylenediacetic acid are flexible ligands and can therefore adopt different conformations in the crystal structure. 1,4-Phenylendiacetic acid is used as a readily available ligand that can coordinate two or more metal ions in bridging-mode, forming extended molecular networks (Pan et al., 2003; Chen et al., 2010a,b,c). The different coordination modes (Chen et al., 2010a,b,c; Rusinek et al., 2013; Ren et al., 2011; Pan et al., 2003; Singha et al., 2014; Singha et al., 2015) of the ligand with lanthanide ions that have been reported to date are shown in Fig. 1.

In this paper we report the synthesis and the crystal structure of a new coordination polymer with chemical formula $\left[\mathrm{La}_{2}(p \text {-pda })_{3}\left(\mathrm{H}_{2} \mathrm{O}\right)_{4} \cdot 8 \mathrm{H}_{2} \mathrm{O}\right]_{\infty}$.

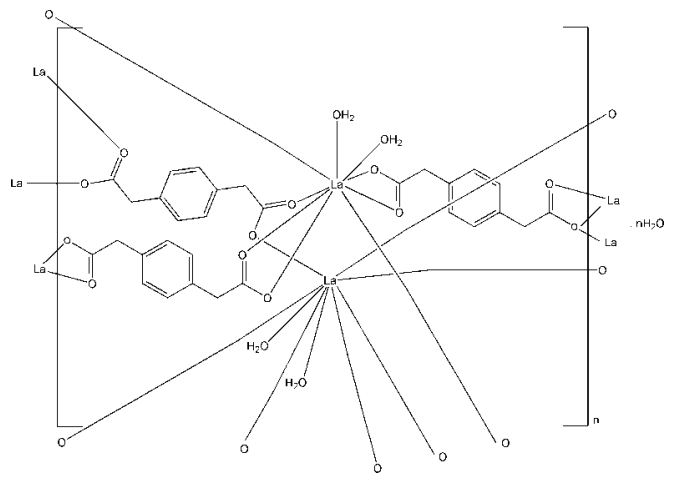

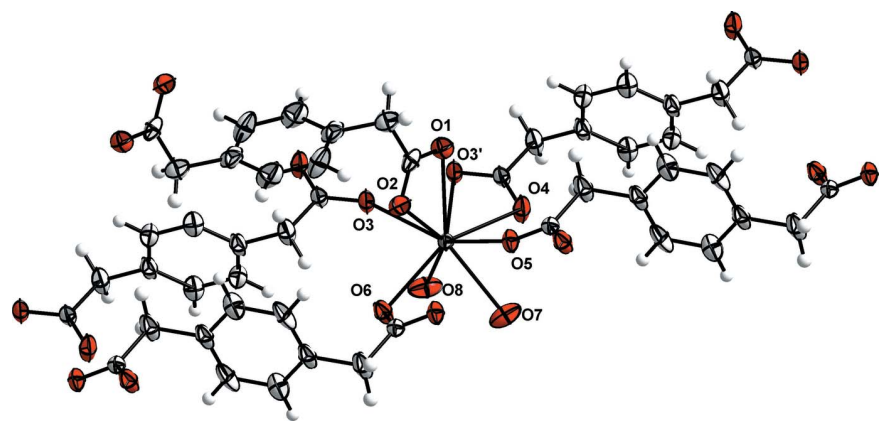

Figure 2

Coordination environment of $\mathrm{La}^{3+}$ in $\left[\mathrm{La}_{2}(p-\text { pda })_{3}\left(\mathrm{H}_{2} \mathrm{O}\right)_{4} \cdot 8 \mathrm{H}_{2} \mathrm{O}\right]_{\infty}$. Symmetry code: (') $2-x, 1-y, 1-z$. Hydrogen atoms of the water molecules have been omitted for clarity.

\section{Structural commentary}

The crystallographically independent $\mathrm{La}^{3+}$ ion is nona-coordinated by seven oxygen atoms $\left.(\mathrm{O} 1, \mathrm{O} 2, \mathrm{O} 3, \mathrm{O} 4, \mathrm{O} 5, \mathrm{O} 6, \mathrm{O} 3)^{\prime}\right)$ from five $p$ - $\mathrm{pda}^{2-}$ ligands and two oxygen atoms (O8 and $\mathrm{O} 7$ ) from the coordinating water molecules (Fig. 2). The coordination polyhedron can be described as a monocapped distorted square antiprism with atom $\mathrm{O}^{\prime}$ capping the polyhedron [symmetry code: (') $2-x, 1-y, 1-z$ ]. The two square sides of the antiprism are formed by atoms $\mathrm{O} 7, \mathrm{O} 6, \mathrm{O} 2$, $\mathrm{O} 5$ and $\mathrm{O} 8, \mathrm{O} 3, \mathrm{O} 1, \mathrm{O} 4$, respectively. The dihedral angle between the two faces is $5.21(9)^{\circ}$. There are three independent ligands: L1, $\mathbf{L 2}$ and $\mathbf{L 3}$ (Fig. 3). The twisted ligand $\mathbf{L 3}$ exhibits a coordination mode that has never previously been
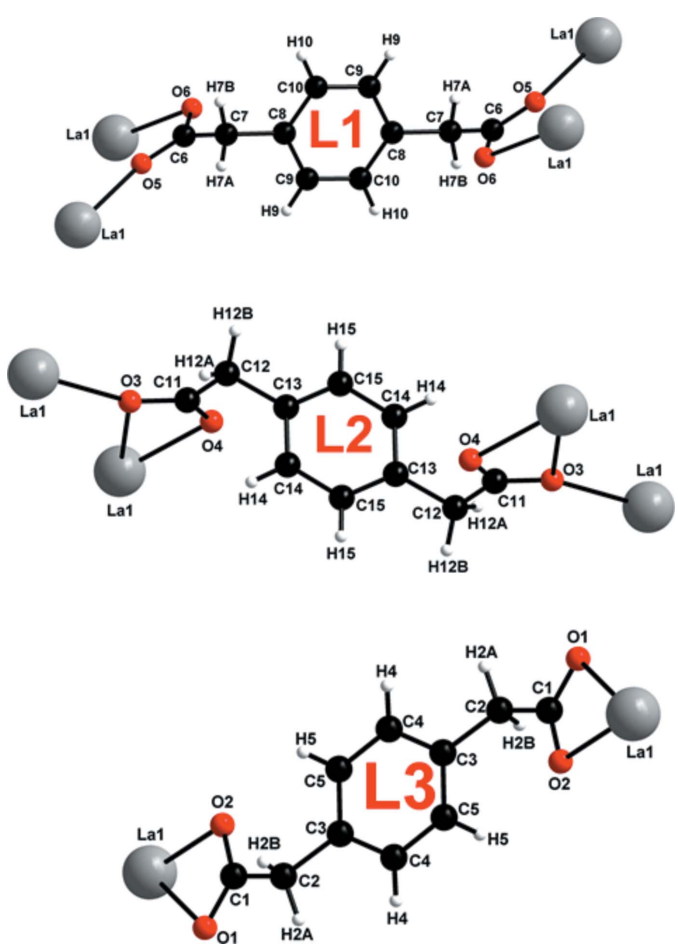

Figure 3

Coordination modes of ligand $\mathbf{L 1}\left(\mu-4\right.$ bis-bidentate mode: $\left(\eta^{1}-\eta^{1}-\mu_{2}\right)$ $\left.\left(\eta^{1}-\eta^{1}-\mu_{2}\right)-\mu_{4}\right), \mathbf{L 2}\left(\mu-4\right.$ bis-tridentate bridging and chelating mode: $\left(\eta^{2}-\right.$ $\left.\left.\eta^{1}-\mu_{2}\right)-\left(\eta^{2}-\eta^{1}-\mu_{2}\right)-\mu_{4}\right)$ and $\mathbf{L 3}\left(\mu-2\right.$ bis-bidentate-chelating mode: $\left(\eta^{1}-\eta^{1}-\right.$ $\left.\left.\left.\mu_{1}\right)-\left(\eta^{1}-\eta^{1}-\mu_{1}\right)-\mu_{2}\right)\right)$ 
Table 1

Hydrogen-bond geometry $\left(\AA,^{\circ}\right)$.

\begin{tabular}{|c|c|c|c|c|}
\hline$D-\mathrm{H} \cdots A$ & $D-\mathrm{H}$ & $\mathrm{H} \cdots A$ & $D \cdots A$ & $D-\mathrm{H} \cdots A$ \\
\hline $\mathrm{O} W 1-\mathrm{H} W 1 A \cdots \mathrm{O} W 1^{\mathrm{iii}}$ & $0.87(9)$ & $2.40(11)$ & $3.067(13)$ & $133(9)$ \\
\hline $\mathrm{O} W 1-\mathrm{H} W 1 B \cdots \mathrm{O} W 4^{\mathrm{iv}}$ & $0.89(9)$ & $2.54(10)$ & $3.298(11)$ & $145(7)$ \\
\hline $\mathrm{O} W 2-\mathrm{H} W 2 A \cdots \mathrm{O} 4^{\mathrm{i}}$ & $0.82(6)$ & $2.10(6)$ & $2.895(5)$ & $164(6)$ \\
\hline $\mathrm{O} W 2-\mathrm{H} W 2 B \cdots \mathrm{O} W 4^{\mathrm{v}}$ & $0.82(6)$ & $2.20(5)$ & $2.855(8)$ & $137(5)$ \\
\hline $\mathrm{O} W 3-\mathrm{H} W 3 A \cdots \mathrm{O} 6^{\mathrm{i}}$ & $0.82(8)$ & $2.02(8)$ & $2.780(8)$ & $154(7)$ \\
\hline $\mathrm{O} W 3-\mathrm{H} W 3 B \cdots \mathrm{O} W 1^{\mathrm{iii}}$ & $0.81(7)$ & $2.40(8)$ & $3.162(11)$ & $156(8)$ \\
\hline $\mathrm{O} W 4-\mathrm{H} W 4 A \cdots \mathrm{O} W 2^{\mathrm{vi}}$ & $0.81(10)$ & $2.49(9)$ & $2.855(8)$ & $109(9)$ \\
\hline $\mathrm{O} 7-\mathrm{H} 7 A \cdots \mathrm{O} 2^{\mathrm{i}}$ & $0.82(4)$ & $1.95(4)$ & $2.741(5)$ & $161(5)$ \\
\hline $\mathrm{O} 7-\mathrm{H} 7 \mathrm{~B} \cdots \mathrm{OW} 4$ & $0.81(5)$ & $2.03(5)$ & $2.800(9)$ & $160(5)$ \\
\hline $\mathrm{O} W 4-\mathrm{H} W 4 B \cdots \mathrm{O} W 3^{\mathrm{vii}}$ & $0.84(9)$ & $2.11(10)$ & $2.824(11)$ & $143(8)$ \\
\hline $\mathrm{O} 8-\mathrm{H} 8 A \cdots \mathrm{O} W 3^{\mathrm{i}}$ & $0.82(4)$ & $2.38(4)$ & $3.175(8)$ & $165(4)$ \\
\hline $\mathrm{O} 8-\mathrm{H} 8 B \cdots \mathrm{O} 1^{\mathrm{ii}}$ & $0.83(4)$ & $1.92(4)$ & $2.725(5)$ & $163(5)$ \\
\hline $\mathrm{C} 7-\mathrm{H} 7 D \cdots \mathrm{O} 4^{\mathrm{i}}$ & 0.97 & 2.54 & $3.442(6)$ & 154 \\
\hline $\mathrm{C} 12-\mathrm{H} 12 B \cdots \mathrm{O} 6^{\mathrm{ii}}$ & 0.97 & 2.51 & $3.406(6)$ & 154 \\
\hline
\end{tabular}

Symmetry codes: (i) $-x+1,-y+1,-z+1$; (ii) $-x+2,-y+1,-z+1$; $\quad$ (iii) $-x+1,-y,-z+1$; (iv) $x, y-1, z ;$ (v) $x, y, z+1$; (vi) $x, y, z-1$; (vii) $x+1, y+1, z$

observed in lanthanide-based coordination polymers involving the $p$-pda ${ }^{2-}$ ligand.

The monocapped square antiprisms are connected to each other by alternating $\mathbf{L 1}$ bridging carboxylate oxygen atoms (O5 and O6) and edge-sharing polyhedra through $\mathbf{L 2}$ oxygen atoms (O3), forming molecular chains along the $a$-axis direction (Fig. 4). These chains are connected to each other through
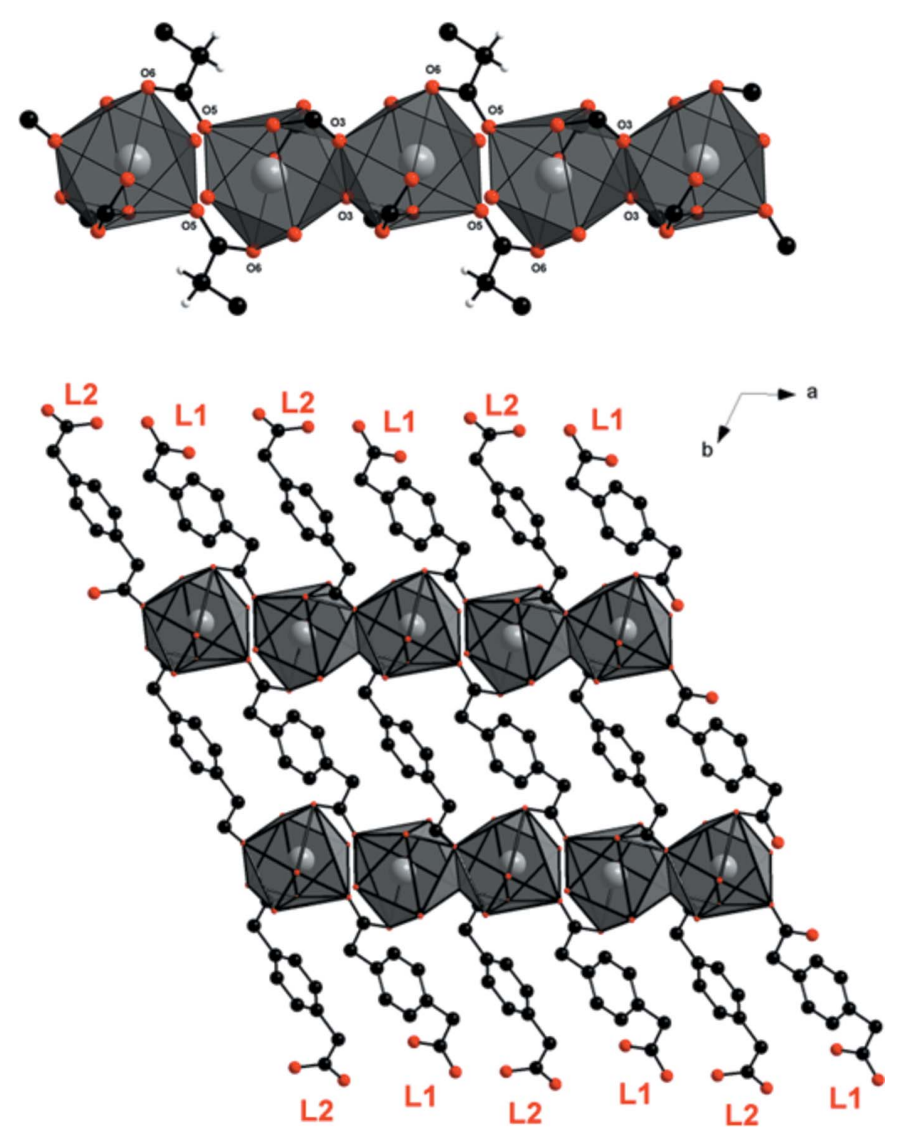

Figure 4

(Top) Projection view of a molecular chain extending parallel to the $a$ axis. (Bottom) Projection view along the $c$ axis of the of the twodimensional molecular network of $\left[\mathrm{La}_{2}(p \text {-pda })_{3}\left(\mathrm{H}_{2} \mathrm{O}\right)_{4} \cdot 8 \mathrm{H}_{2} \mathrm{O}\right]_{\infty}$. Hydrogen atoms have been omitted for clarity.

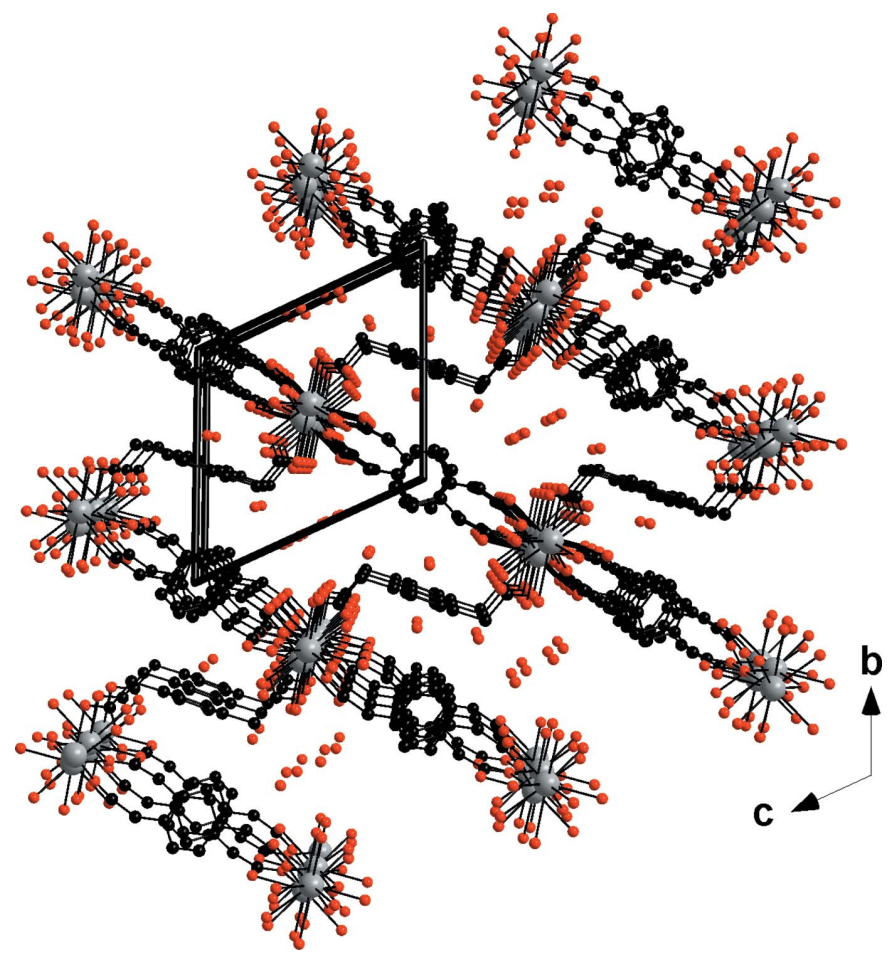

Figure 5

Perspective view along the $a$ axis of $\left[\mathrm{La}_{2}(p-\text { pda })_{3}\left(\mathrm{H}_{2} \mathrm{O}\right)_{4} \cdot 8 \mathrm{H}_{2} \mathrm{O}\right]_{\infty}$. Hydrogen atoms have been omitted for clarity.

ligands $\mathbf{L 1}$ and $\mathbf{L 2}$, which play the role of spacers, forming molecular layers that extend parallel to the $a b$ plane (Fig. 4).

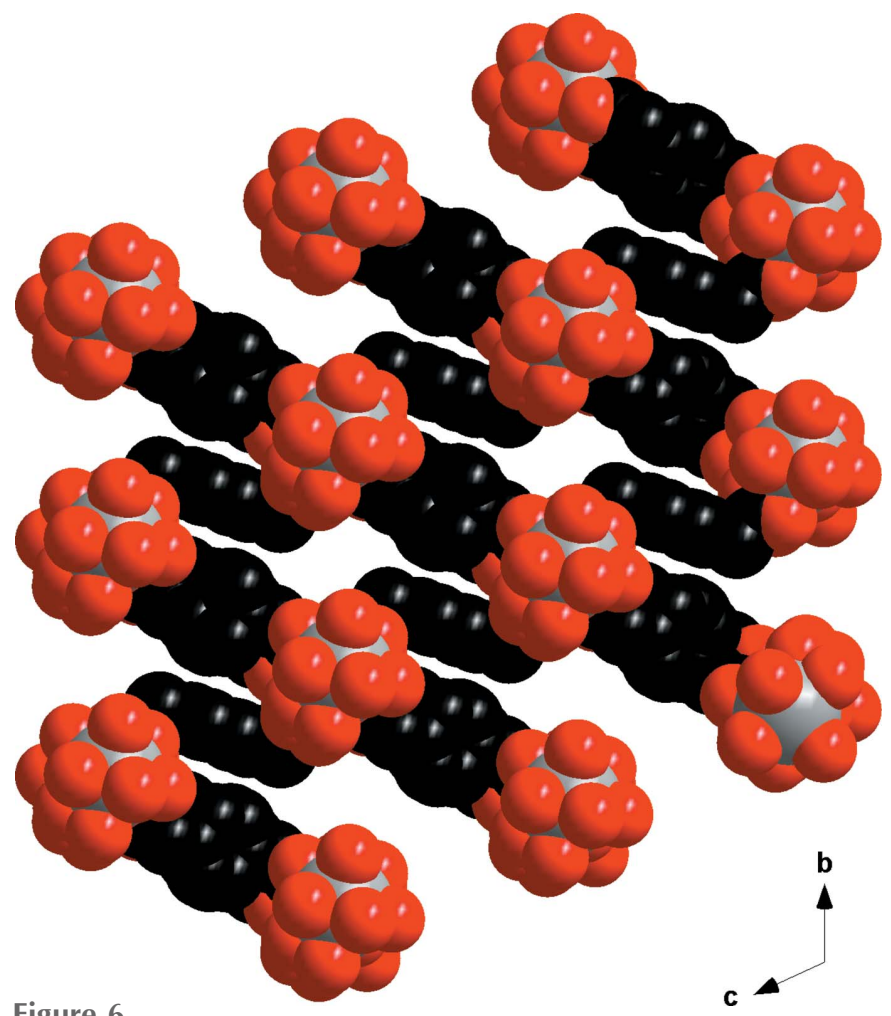

Figure 6

Projection view along the $a$ axis of the molecular skeleton of $\left[\mathrm{La}_{2}(p-\right.$ pda $\left.)_{3}\left(\mathrm{H}_{2} \mathrm{O}\right)_{4} \cdot 8 \mathrm{H}_{2} \mathrm{O}\right]_{\infty}$ in space-filling mode. Hydrogen atoms and crystallization water molecules have been omitted. 
These layers are further connected through the twisted ligand L3, leading to a three-dimensional molecular framework (Fig. 5). Ligand $\mathbf{L 3}$ acts as a spacer between the different polymeric layers because of its anti-anti conformation.

The framework has channels along the $a$-axis direction in which the water molecules of crystallization are located. They are bound to the molecular skeleton via a hydrogen-bonded network (Table 1). As can be seen from Fig. 6, the threedimensional crystal structure could potentially present some porosity properties. Indeed, removal of the water molecules of crystallization could create empty channels, as has been reported previously (Kustaryono et al., 2010; Kerbellec et al., 2008). For the coordination polymer in this study, the potential porosity is calculated to be $750(20) \mathrm{m}^{2} \mathrm{~g}^{-1}$ for $\mathrm{N}_{2}$ with a kinetic radius of $1.83 \AA$. The calculation was performed using a method described elsewhere (Kustaryono et al., 2010; Kerbellec et al., 2008).

Other crystal structures of lanthanide coordination polymers with the $p-\mathrm{pda}^{2-}$ ligand have been reported previously. This series of compounds, first described by Pan et al. (2003) has been widely studied because of potential applications in various fields such as explosives detection (Singha et al., 2014, 2015), gas sorption (Pan et al., 2003) or catalysis (Ren et al., 2011). These compounds, with general chemical formula $\left[L n_{2}(p \text {-pda })_{3}\left(\mathrm{H}_{2} \mathrm{O}\right) \cdot 2 \mathrm{H}_{2} \mathrm{O}\right]_{\infty}$ with $L n=\mathrm{La}-\mathrm{Ho}$ have been obtained by hydrothermal synthesis and therefore present a lower hydration rate and a higher density than $\left[\mathrm{La}_{2}(p-\text { pda })_{3^{-}}\right.$ $\left.\left(\mathrm{H}_{2} \mathrm{O}\right)_{4} \cdot 8 \mathrm{H}_{2} \mathrm{O}\right]_{\infty}\left\{D_{\text {calc }}=1871 \mathrm{~g} \mathrm{~cm}^{-3}\right.$ for $\left[\operatorname{Ln}_{2}(\mathrm{p}-\mathrm{pda})_{3}\left(\mathrm{H}_{2} \mathrm{O}\right) \cdot-\right.$ $\left.2 \mathrm{H}_{2} \mathrm{O}\right]_{\infty}$. Their three-dimensional crystal structures can be described on the basis of helicoidal molecular chains linked by $p$-pda ${ }^{2-}$ ligands.

The luminescent and porosity properties of these compounds are interesting, which suggests that the physical properties of compounds isostructural to $\left[\mathrm{La}_{2}(p\right.$ pda $\left.)_{3}\left(\mathrm{H}_{2} \mathrm{O}\right)_{4} \cdot 8 \mathrm{H}_{2} \mathrm{O}\right]_{\infty}$ and involving other lanthanide ions (lanthanum is a diamagnetic non-luminescent ion) would be worth studying. Unfortunately, despite great synthetic efforts, no such compound has been obtained to date.

The compound reported here was obtained by crystallization in a gel (see next section; Luo et al., 2013), and as such is the first result from our group related to lanthanide-based coordination polymers with 1,4-phenylenediacetate ligands.

\section{Synthesis and crystallization}

Lanthanum oxide $\left(\mathrm{La}_{2} \mathrm{O}_{3}\right)$ was suspended in a small quantity of water. The suspension was then brought to about $323 \mathrm{~K}$ and concentrated hydrochloric acid was added dropwise under magnetic stirring, until a clear solution was obtained. The solution was then evaporated to dryness and the resulting solid was dissolved in absolute ethanol for removal of the residual hydrochloric acid. Crystallization of the salt was then obtained by adding diethyl ether $\left(\mathrm{Et}_{2} \mathrm{O}\right)$. The obtained microcrystalline solid was filtered and dried in the open air. The product $\mathrm{LaCl}_{3} \cdot 7 \mathrm{H}_{2} \mathrm{O}$ was obtained in close to $100 \%$ yield.

1,4-Phenylenediacetic acid, $\mathrm{H}_{2}$ (p-pda), was purchased from Sigma-Aldrich and used without further purification. Its
Table 2

Experimental details.

\begin{tabular}{|c|c|}
\hline \multicolumn{2}{|l|}{ Crystal data } \\
\hline Chemical formula & {$\left[\mathrm{La}_{2}\left(\mathrm{C}_{10} \mathrm{H}_{8} \mathrm{O}_{4}\right)_{3}\left(\mathrm{H}_{2} \mathrm{O}\right)_{4}\right] \cdot 8 \mathrm{H}_{2} \mathrm{O}$} \\
\hline$M_{\mathrm{r}}$ & 1070.05 \\
\hline Crystal system, space group & Triclinic, $P \overline{1}$ \\
\hline Temperature (K) & 293 \\
\hline$a, b, c(\AA)$ & $9.1197(2), 11.1231(2), 11.9434(2)$ \\
\hline$\alpha, \beta, \gamma\left({ }^{\circ}\right)$ & $\begin{array}{l}107.049(1), 107.729(1) \\
\quad 106.622(1)\end{array}$ \\
\hline$V\left(\AA^{3}\right)$ & $1005.21(3)$ \\
\hline$Z$ & 1 \\
\hline Radiation type & Mo $K \alpha$ \\
\hline$\mu\left(\mathrm{mm}^{-1}\right)$ & 2.18 \\
\hline Crystal size $(\mathrm{mm})$ & $0.08 \times 0.06 \times 0.05$ \\
\hline
\end{tabular}

Data collection

Diffractometer

No. of measured, independent and observed $[I>2 \sigma(I)]$ reflections

$R_{\text {int }}$

$(\sin \theta / \lambda)_{\max }\left(\AA^{-1}\right)$

Nonius KappaCCD
$4588,4588,3751$

0.045
0.649

$0.030,0.068,1.03$
4588
283
18
$\mathrm{H}$ atoms treated by a mixture of
$\quad$ independent and constrained
$\quad$ refinement
$1.76,-0.65$

Refinement

$R\left[F^{2}>2 \sigma\left(F^{2}\right)\right], w R\left(F^{2}\right), S$

No. of reflections

No. of parameters

No. of restraints

$\mathrm{H}$-atom treatment

$\Delta \rho_{\max }, \Delta \rho_{\min }\left(\mathrm{e} \AA^{-3}\right)$ $1.76,-0.65$

Computer programs: COLLECT (Bruker, 2004), DENZO and SCALEPACK (Otwinowski \& Minor, 1997), SHELXT (Sheldrick, 2015a), SHELXL (Sheldrick, 2015b), DIAMOND (Brandenburg, 2001), Win GX (Farrugia, 2012).

disodium salt was prepared by addition of two equivalents of sodium hydroxide to a suspension of the acid in de-ionized water. The obtained clear solution was evaporated to dryness and then refluxed in ethanol for one h. Addition of diethyl ether provoked precipitation of $\mathrm{Na}_{2}(p$-pda) in $90 \%$ yield. UVvis absorption spectrum of a $4.3 \times 10^{-4} \mathrm{~mol} \mathrm{~L}^{-1}$ aqueous solution of the disodium salt of $\mathrm{H}_{2}$ (p-pda) was measured with a Perkin-Elmer Lambda 650 spectrometer equipped with a $60 \mathrm{~mm}$ integrating sphere. It showed a maximum absorption at $225 \mathrm{~nm}$. This short absorption wavelength, compared to other ligands in the literature (Badiane et al., 2017a,b; Freslon et al., 2016; Fan et al., 2015; Badiane et al., 2018), can be related to the $-\mathrm{CH}_{2}$ - groups that cut conjugation.

Single crystals of the coordination polymer were obtained by slow diffusion of dilute aqueous solutions of lanthanum chloride $(0.25 \mathrm{mmol}$ in $10 \mathrm{~mL})$ and of the sodium salt of paraphenylenediacetate $(0.25 \mathrm{mmol}$ in $10 \mathrm{~mL})$ through an agaragar gel in a U-shaped tube. The gel was purchased from Acros Organics and jellified according to established procedures (Henisch, 1988; Daiguebonne et al., 2003). After several weeks, prismatic single crystals were obtained.

\section{Refinement}

Crystal data, data collection and structure refinement details are summarized in Table 2. Hydrogen atoms bound to the organic ligands were placed at idealized positions $(\mathrm{C}-\mathrm{H}=$ $0.93-0.97 \AA$ ) and refined as riding with $U_{\text {iso }}(\mathrm{H})=1.2 U_{\text {eq }}(\mathrm{C})$. 
The water hydrogen atoms were localized and constrained. The thermal agitation of the two water molecules of crystallization was constrained. In order to stabilize the refinement several restraints (DANG, DFIX) were used for the hydrogen atoms bound to water oxygens.

\section{Acknowledgements}

The French Cooperation Agency in Senegal is acknowledged for financial support.

\section{References}

Badiane, A.-M., Freslon, S., Daiguebonne, C., Suffren, Y., Bernot, K., Calvez, G., Costuas, K., Camara, M. \& Guillou, O. (2018). Inorg. Chem. 57, 3399-3410.

Badiane, I., Freslon, S., Suffren, Y., Daiguebonne, C., Calvez, G., Bernot, K., Camara, M. \& Guillou, O. (2017a). Cryst. Growth Des. 17, 1224-1234.

Badiane, I., Freslon, S., Suffren, Y., Daiguebonne, C., Calvez, G., Bernot, K., Camara, M. \& Guillou, O. (2017b). Inorg. Chim. Acta, 461, 136-144.

Binnemans, K. (2009). Chem. Rev. 109, 4283-4374.

Brandenburg, K. (2001). DIAMOND. Crystal Impact GbR, Bonn, Germany.

Calvez, G., Bernot, K., Guillou, O., Daiguebonne, C., Caneschi, A. \& Mahé, N. (2008). Inorg. Chim. Acta, 361, 3997-4003.

Chen, Z., Xiong, W., Zhang, Z. \& Liang, F. (2010a). Z. Anorg. Allg. Chem. 636, 1392-1396.

Chen, Z., Xiong, W., Zhang, Z., Liang, F. P. \& Luo, B. C. (2010b). Transition Met. Chem. 35, 991-997.

Chen, Z., Zhao, Q., Xiong, W., Zhang, Z. \& Liang, F. (2010c). Z. Anorg. Allg. Chem. 636, 2691-2697.

Cui, Y., Yue, Y., Qian, G. \& Chen, B. (2012). Chem. Rev. 112, 11261162.

Daiguebonne, C., Deluzet, A., Camara, M., Boubekeur, K., Audebrand, N., Gérault, Y., Baux, C. \& Guillou, O. (2003). Cryst. Growth Des. 3, 1015-1020.

Daiguebonne, C., Kerbellec, N., Guillou, O., Bünzli, J. C., Gumy, F., Catala, L., Mallah, T., Audebrand, N., Gérault, Y., Bernot, K. \& Calvez, G. (2008). Inorg. Chem. 47, 3700-3708.

Eddaoudi, M., Kim, J., Rosi, N., Vodak, D., Wachter, J., O'Keeffe, M. \& Yaghi, O. M. (2002). Science, 295, 469-472.

Fabelo, O., Cañadillas-Delgado, L., Pasán, J., Delgado, F. S., Lloret, F., Cano, J., Julve, M. \& Ruiz-Pérez, C. (2009a). Inorg. Chem. 48, 11342-11351.

Fabelo, O., Pasán, J., Cañadillas-Delgado, L., Delgado, F. S., Lloret, F., Julve, M. \& Ruiz-Pérez, C. (2009b). Inorg. Chem. 48, 6086-6095.

Fan, X., Freslon, S., Daiguebonne, C., Calvez, G., Le Pollès, L., Bernot, K. \& Guillou, O. (2014). J. Mater. Chem. C. 2, 5510-5525.

Fan, X., Freslon, S., Daiguebonne, C., Pollès, L. L., Calvez, G., Bernot, K., Yi, X., Huang, G. \& Guillou, O. (2015). Inorg. Chem. 54, 55345546.

Farrugia, L. J. (2012). J. Appl. Cryst. 45, 849-854.

Freslon, S., Luo, Y., Calvez, G., Daiguebonne, C., Guillou, O., Bernot, K., Michel, V. \& Fan, X. (2014). Inorg. Chem. 53, 1217-1228.
Freslon, S., Luo, Y., Daiguebonne, C., Calvez, G., Bernot, K. \& Guillou, O. (2016). Inorg. Chem. 55, 794-802.

Hamon, L., Llewellyn, P. L., Devic, T., Ghoufi, A., Clet, G., Guillerm, V., Pirngruber, G. D., Maurin, G., Serre, C., Driver, G., van Beek, W., Jolimaître, E., Vimont, A., Daturi, M. \& Férey, G. (2009). J. Am. Chem. Soc. 131, 17490-17499.

Henisch, H. K. (1988). Crystals in Gels and Liesegang Rings; Cambridge University Press: Cambridge.

Huang, Y. G., Jiang, F. L., Yuan, D. Q., Wu, M. Y., Gao, Q., Wei, W. \& Hong, M. C. (2009). J. Solid State Chem. 182, 215-222.

Kerbellec, N., Daiguebonne, C., Bernot, K., Guillou, O. \& Le Guillou, X. (2008). J. Alloys Compd. 451, 377-383.

Kustaryono, D., Kerbellec, N., Calvez, G., Freslon, S., Daiguebonne, C. \& Guillou, O. (2010). Cryst. Growth Des. 10, 775-781.

Lee, J., Farha, O. K., Roberts, J., Scheidt, K. A., Nguyen, S., inh, T. \& Hupp, J. T. (2009). Chem. Soc. Rev. 38, 1450-1459.

Li, D. S., Zhang, M. L., Zhao, J., Wang, D. J., Zhang, P., Wang, N. \& Wang, Y. Y. (2009). Inorg. Chem. Commun. 12, 1027-1030.

Luo, F., Batten, S. R., Che, Y. \& Zheng, J. M. (2007). Chem. Eur. J. 13, 4948-4955.

Luo, Y., Bernot, K., Calvez, G., Freslon, S., Daiguebonne, C., Guillou, O., Kerbellec, N. \& Roisnel, T. (2013). CrystEngComm, 15, 1882 1896.

Luo, Y., Calvez, G., Freslon, S., Bernot, K., Daiguebonne, C. \& Guillou, O. (2011a). Eur. J. Inorg. Chem. 3705-3716.

Luo, Y., Calvez, G., Freslon, S., Daiguebonne, C., Roisnel, T. \& Guillou, O. (2011b). Inorg. Chim. Acta, 368, 170-178.

Otwinowski, Z. \& Minor, W. (1997). Methods in Enzymology, Vol. 276, Macromolecular Crystallography, Part A, edited by C. W. Carter Jr \& R. M. Sweet, pp. 307-326. New York: Academic Press.

Pan, L., Adams, K. M., Hernandez, H. E., Wang, X., Zheng, Ch., Hattori, Y. \& Kaneko, K. (2003). J. Am. Chem. Soc. 125, 30623067.

Qiu, Y., Daiguebonne, C., Liu, J., Zeng, R., Kerbellec, N., Deng, H. \& Guillou, O. (2007). Inorg. Chim. Acta, 360, 3265-3271.

Reineke, T. M., Eddaoudi, M., O'Keeffe, M. \& Yaghi, O. M. (1999). Angew. Chem. Int. Ed. 38, 2590-2594.

Ren, Y.-W., Liang, J.-X., Lu, J.-X., Cai, B.-W., Shi, D.-B., Qi, C.-R., Jiang, H.-F., Chen, J. \& Zheng, D. (2011). Eur. J. Inorg. Chem. pp. 4369-4376.

Rusinek, I., Sienkiewicz-Gromiuk, J., Mazur, L. \& Rzączyńska, Z. (2013). J. Inorg. Organomet. Polym. 23, 1068-1077.

Sessoli, R. \& Powell, A. K. (2009). Coord. Chem. Rev. 253, 2328-2341.

Sheldrick, G. M. (2015a). Acta Cryst. A71, 3-8.

Sheldrick, G. M. (2015b). Acta Cryst. C71, 3-8.

Singha, D.-K., Bhattacharya, S., Majee, P., Mondal, S.-K., Kumar, M. \& Mahata, P. (2014). J. Mater. Chem. A, 2, 20908-20915.

Singha, D.-K., Majee, P., Mondal, S. K. \& Mahata, P. (2015). Eur. J. Inorg. Chem. pp. 1390-1397.

Singh, W. M. \& Baruah, J. B. (2009). Inorg. Chim. Acta, 362, 42684271.

Uebler, J. W. \& LaDuca, R. L. (2012). Inorg. Chem. Commun. 19, 3135.

Wang, L., Song, T., Li, C., Xia, J., Wang, S., Wang, L. \& Xu, J. (2012). J. Solid State Chem. 190, 208-215.

Xin, L. Y., Liu, G. Z. \& Wang, L. Y. (2011). J. Solid State Chem. 184, 1387-1392. 


\section{supporting information}

Acta Cryst. (2019). E75, 378-382 [https://doi.org/10.1107/S2056989019002378]

Synthesis and crystal structure of a new coordination polymer based on lanthanum and 1,4-phenylenediacetate ligands

\section{Magatte Camara, Insa Badiane, Mamoudou Diallo, Carole Daiguebonne and Olivier Guillou}

Computing details

Data collection: COLLECT (Bruker, 2004); cell refinement: SCALEPACK (Otwinowski \& Minor, 1997); data reduction: DENZO and SCALEPACK (Otwinowski \& Minor, 1997); program(s) used to solve structure: SHELXT (Sheldrick, 2015a); program(s) used to refine structure: SHELXL (Sheldrick, 2015b); molecular graphics: DIAMOND (Brandenburg, 2001); software used to prepare material for publication: WinGX (Farrugia, 2012).

Poly[[tetraaquatris( $\mu$-1,4-phenylenediacetato)dilanthanum(III)] octahydrate]

\section{Crystal data}

$\left[\mathrm{La}_{2}\left(\mathrm{C}_{10} \mathrm{H}_{8} \mathrm{O}_{4}\right)_{3}\left(\mathrm{H}_{2} \mathrm{O}\right)_{4}\right] \cdot 8 \mathrm{H}_{2} \mathrm{O}$

$M_{r}=1070.05$

Triclinic, $P \overline{1}$

Hall symbol: -P 1

$a=9.1197(2) \AA$

$b=11.1231(2) \AA$

$c=11.9434(2) \AA$

$\alpha=107.049(1)^{\circ}$

$\beta=107.729(1)^{\circ}$

$\gamma=106.622(1)^{\circ}$

$V=1005.21(3) \AA^{3}$

Data collection

Nonius KappaCCD diffractometer

Radiation source: Enraf Nonius FR590

Horizonally mounted graphite crystal monochromator

Detector resolution: 9 pixels $\mathrm{mm}^{-1}$

CCD rotation images, thick slices scans

4588 measured reflections

\section{Refinement}

Refinement on $F^{2}$

Least-squares matrix: full

$R\left[F^{2}>2 \sigma\left(F^{2}\right)\right]=0.030$

$w R\left(F^{2}\right)=0.068$

$S=1.03$

4588 reflections

283 parameters

18 restraints
$Z=1$

$F(000)=534$

$D_{\mathrm{x}}=1.768 \mathrm{Mg} \mathrm{m}^{-3}$

Mo $K \alpha$ radiation, $\lambda=0.71073 \AA$

Cell parameters from 15558 reflections

$\theta=2.9-27.5^{\circ}$

$\mu=2.18 \mathrm{~mm}^{-1}$

$T=293 \mathrm{~K}$

Prism, colorless

$0.08 \times 0.06 \times 0.05 \mathrm{~mm}$

4588 independent reflections

3751 reflections with $I>2 \sigma(I)$

$R_{\text {int }}=0.045$

$\theta_{\max }=27.5^{\circ}, \theta_{\min }=3.6^{\circ}$

$h=-10 \rightarrow 11$

$k=-14 \rightarrow 14$

$l=-15 \rightarrow 15$

0 constraints

Primary atom site location: dual

Secondary atom site location: dual

Hydrogen site location: mixed

$\mathrm{H}$ atoms treated by a mixture of independent and constrained refinement

$w=1 /\left[\sigma^{2}\left(F_{\mathrm{o}}^{2}\right)+(0.0308 P)^{2}\right]$

where $P=\left(F_{\mathrm{o}}^{2}+2 F_{\mathrm{c}}^{2}\right) / 3$ 
$(\Delta / \sigma)_{\max }=0.001$

$\Delta \rho_{\min }=-0.65$ e $\AA^{-3}$

$\Delta \rho_{\max }=1.76$ e $\AA^{-3}$

Special details

Geometry. All esds (except the esd in the dihedral angle between two 1.s. planes) are estimated using the full covariance matrix. The cell esds are taken into account individually in the estimation of esds in distances, angles and torsion angles; correlations between esds in cell parameters are only used when they are defined by crystal symmetry. An approximate (isotropic) treatment of cell esds is used for estimating esds involving l.s. planes.

Fractional atomic coordinates and isotropic or equivalent isotropic displacement parameters $\left(\AA^{2}\right)$

\begin{tabular}{|c|c|c|c|c|}
\hline & $x$ & $y$ & $z$ & $U_{\text {iso }} * / U_{\text {eq }}$ \\
\hline La1 & $0.75090(2)$ & $0.52031(2)$ & $0.47969(2)$ & $0.02048(7)$ \\
\hline O5 & $0.4472(3)$ & $0.3677(2)$ & $0.4069(2)$ & $0.0332(6)$ \\
\hline $\mathrm{O} 3$ & $1.0575(3)$ & $0.5881(3)$ & $0.6253(2)$ & $0.0323(6)$ \\
\hline $\mathrm{O} 1$ & $0.7627(3)$ & $0.3041(3)$ & $0.5311(2)$ & $0.0336(6)$ \\
\hline O6 & $0.8146(3)$ & $0.7579(3)$ & $0.6435(3)$ & $0.0362(6)$ \\
\hline $\mathrm{O} 2$ & $0.7422(3)$ & $0.4674(3)$ & $0.6736(2)$ & $0.0351(6)$ \\
\hline $\mathrm{O} 4$ & $0.6840(3)$ & $0.3616(3)$ & $0.2490(2)$ & $0.0415(7)$ \\
\hline $\mathrm{O} 7$ & $0.5690(4)$ & $0.5882(4)$ & 0.3233 & $0.0486(8)$ \\
\hline $\mathrm{H} 7 \mathrm{~A}$ & 0.468 & $0.558(4)$ & $0.307(5)$ & $0.058^{*}$ \\
\hline H7B & $0.591(5)$ & $0.651(4)$ & $0.302(5)$ & $0.058^{*}$ \\
\hline O8 & $0.9316(4)$ & $0.7051(3)$ & $0.4326(4)$ & $0.0503(8)$ \\
\hline H8A & $0.947(6)$ & 0.785 & $0.470(4)$ & $0.060^{*}$ \\
\hline H8B & $1.026(4)$ & $0.712(4)$ & $0.435(5)$ & $0.060 *$ \\
\hline C6 & $0.6834(4)$ & $0.7426(3)$ & $0.6621(3)$ & $0.0260(7)$ \\
\hline C11 & $0.8165(4)$ & $0.3436(3)$ & $0.2659(3)$ & $0.0261(7)$ \\
\hline $\mathrm{C} 1$ & $0.7640(4)$ & $0.3589(4)$ & $0.6405(4)$ & $0.0315(8)$ \\
\hline $\mathrm{C} 13$ & $0.6579(5)$ & $0.1147(4)$ & 0.0739 & $0.0340(9)$ \\
\hline $\mathrm{C} 12$ & $0.8247(5)$ & $0.2382(4)$ & 0.1565 (4) & $0.0390(9)$ \\
\hline $\mathrm{H} 12 \mathrm{~A}$ & 0.857342 & 0.281262 & 0.102898 & $0.058^{*}$ \\
\hline H12B & 0.910563 & 0.207990 & 0.192279 & $0.058^{*}$ \\
\hline C14 & $0.6017(5)$ & $0.0190(4)$ & 0.1196 & $0.0427(10)$ \\
\hline H14 & 0.669560 & 0.030195 & 0.201149 & $0.051^{*}$ \\
\hline $\mathrm{C} 8$ & $0.8472(5)$ & $0.9325(4)$ & $0.8881(4)$ & $0.0340(9)$ \\
\hline $\mathrm{C} 10$ & $0.9564(5)$ & $1.0646(4)$ & $0.9217(4)$ & $0.0433(10)$ \\
\hline H10 & 0.928413 & 1.109955 & 0.869797 & $0.052^{*}$ \\
\hline $\mathrm{C} 15$ & $0.5547(5)$ & $0.0946(4)$ & $-0.0469(4)$ & $0.0412(10)$ \\
\hline H15 & 0.589046 & 0.157191 & -0.080797 & $0.049^{*}$ \\
\hline $\mathrm{C} 7$ & $0.6832(5)$ & $0.8578(4)$ & 0.7675 & $0.0445(11)$ \\
\hline $\mathrm{H} 7 \mathrm{C}$ & 0.660724 & 0.923722 & 0.734480 & $0.067^{*}$ \\
\hline H7D & 0.592152 & 0.819798 & 0.789973 & $0.067^{*}$ \\
\hline C9 & $0.8927(6)$ & $0.8687(4)$ & 0.9685 (4) & $0.0461(11)$ \\
\hline H9 & 0.820475 & 0.779508 & 0.948162 & $0.055^{*}$ \\
\hline $\mathrm{C} 3$ & $0.8997(5)$ & $0.3972(4)$ & $0.8732(4)$ & $0.0392(9)$ \\
\hline $\mathrm{C} 4$ & $1.0738(6)$ & $0.4427(5)$ & $0.9267(4)$ & $0.0514(11)$ \\
\hline $\mathrm{H} 4$ & 1.125405 & 0.405378 & 0.878263 & $0.062^{*}$ \\
\hline $\mathrm{C} 5$ & $0.8281(6)$ & $0.4561(5)$ & $0.9477(4)$ & $0.0501(11)$ \\
\hline H5 & 0.711419 & 0.427472 & 0.913133 & $0.060^{*}$ \\
\hline
\end{tabular}




$\begin{array}{lllll}\text { C2 } & 0.7931(6) & 0.2928(4) & 0.7347(4) & 0.0460(10) \\ \text { H2A } & 0.684726 & 0.235879 & 0.727251 & 0.069^{*} \\ \text { H2B } & 0.848359 & 0.233001 & 0.711571 & 0.069^{*} \\ \text { OW1 } & 0.6313(10) & 0.0072(5) & 0.4440(6) & 0.1327(17) \\ \text { HW1A } & 0.589(11) & -0.025(9) & 0.491(8) & 0.159^{*} \\ \text { HW1B } & 0.639(13) & -0.072(6) & 0.411(8) & 0.159^{*} \\ \text { OW2 } & 0.5351(5) & 0.6185(4) & 0.9718(4) & 0.0741(11) \\ \text { HW2A } & 0.476(7) & 0.639(6) & 0.920(5) & 0.111^{*} \\ \text { HW2B } & 0.622(6) & 0.678(5) & 1.033(5) & 0.111^{*} \\ \text { OW3 } & 0.0357(9) & 0.0056(6) & 0.3870(6) & 0.1327(17) \\ \text { HW3A } & 0.059(10) & 0.059(7) & 0.354(7) & 0.159^{*} \\ \text { HW3B } & 0.122(7) & 0.023(10) & 0.447(6) & 0.159^{*} \\ \text { OW4 } & 0.7105(10) & 0.7873(7) & 0.2433(6) & 0.154(3) \\ \text { HW4A } & 0.649(10) & 0.805(11) & 0.192(8) & 0.185^{*} \\ \text { HW4B } & 0.810(5) & 0.831(11) & 0.255(10) & 0.185^{*}\end{array}$

Atomic displacement parameters $\left(\AA^{2}\right)$

\begin{tabular}{|c|c|c|c|c|c|c|}
\hline & $U^{11}$ & $U^{22}$ & $U^{33}$ & $U^{12}$ & $U^{13}$ & $U^{23}$ \\
\hline La1 & $0.01729(10)$ & $0.02001(10)$ & $0.01850(10)$ & $0.00633(7)$ & $0.00482(7)$ & $0.00498(7)$ \\
\hline $\mathrm{O} 5$ & $0.0242(13)$ & $0.0258(13)$ & $0.0312(13)$ & $0.0032(10)$ & $0.0034(11)$ & $0.0040(11)$ \\
\hline $\mathrm{O} 3$ & $0.0227(13)$ & $0.0340(14)$ & $0.0229(12)$ & $0.0074(11)$ & $0.0027(10)$ & $0.0011(11)$ \\
\hline O1 & $0.0352(14)$ & $0.0353(14)$ & $0.0339(14)$ & $0.0161(12)$ & $0.0168(12)$ & $0.0155(12)$ \\
\hline O6 & $0.0247(13)$ & $0.0283(13)$ & $0.0415(15)$ & $0.0089(10)$ & $0.0129(12)$ & $-0.0005(11)$ \\
\hline $\mathrm{O} 2$ & $0.0403(15)$ & $0.0427(15)$ & $0.0332(14)$ & $0.0254(13)$ & $0.0173(12)$ & $0.0200(12)$ \\
\hline $\mathrm{O} 4$ & $0.0267(14)$ & $0.0515(17)$ & $0.0253(13)$ & $0.0175(12)$ & $0.0040(11)$ & $-0.0052(12)$ \\
\hline $\mathrm{O} 7$ & $0.0425(17)$ & $0.075(2)$ & $0.067(2)$ & $0.0390(17)$ & $0.0345(17)$ & $0.0532(18)$ \\
\hline O8 & $0.0456(18)$ & $0.0523(18)$ & $0.088(2)$ & $0.0315(16)$ & $0.0434(18)$ & $0.0469(19)$ \\
\hline C6 & $0.0228(17)$ & $0.0219(17)$ & $0.0260(17)$ & $0.0100(14)$ & $0.0051(14)$ & $0.0052(14)$ \\
\hline C11 & $0.0187(16)$ & $0.0250(17)$ & $0.0236(16)$ & $0.0060(13)$ & $0.0062(14)$ & $0.0018(14)$ \\
\hline $\mathrm{C} 1$ & $0.0184(17)$ & $0.041(2)$ & $0.0332(19)$ & $0.0099(15)$ & $0.0083(15)$ & $0.0177(17)$ \\
\hline $\mathrm{C} 13$ & $0.0261(19)$ & $0.030(2)$ & $0.0297(19)$ & $0.0117(16)$ & $0.0070(16)$ & $-0.0041(16)$ \\
\hline C12 & $0.0233(18)$ & $0.040(2)$ & $0.0318(19)$ & $0.0098(16)$ & $0.0074(16)$ & $-0.0057(17)$ \\
\hline C14 & $0.039(2)$ & $0.044(2)$ & $0.028(2)$ & $0.0198(19)$ & $0.0002(18)$ & $0.0047(18)$ \\
\hline $\mathrm{C} 8$ & $0.031(2)$ & $0.0293(19)$ & $0.0290(19)$ & $0.0102(16)$ & $0.0138(16)$ & $-0.0032(16)$ \\
\hline $\mathrm{C} 10$ & $0.042(2)$ & $0.033(2)$ & $0.037(2)$ & $0.0058(18)$ & $0.0114(19)$ & $0.0059(18)$ \\
\hline $\mathrm{C} 15$ & $0.043(2)$ & $0.037(2)$ & $0.031(2)$ & $0.0133(19)$ & $0.0076(18)$ & $0.0078(17)$ \\
\hline C7 & $0.026(2)$ & $0.040(2)$ & $0.043(2)$ & $0.0127(17)$ & $0.0102(18)$ & $-0.0078(19)$ \\
\hline C9 & $0.042(2)$ & $0.0225(19)$ & $0.048(2)$ & $-0.0033(17)$ & $0.016(2)$ & $0.0006(18)$ \\
\hline $\mathrm{C} 3$ & $0.043(2)$ & $0.044(2)$ & $0.035(2)$ & $0.0206(19)$ & $0.0123(18)$ & $0.0260(18)$ \\
\hline $\mathrm{C} 4$ & $0.051(3)$ & $0.073(3)$ & $0.041(2)$ & $0.037(2)$ & $0.022(2)$ & $0.023(2)$ \\
\hline $\mathrm{C} 5$ & $0.032(2)$ & $0.077(3)$ & $0.042(2)$ & $0.024(2)$ & $0.0112(19)$ & $0.027(2)$ \\
\hline $\mathrm{C} 2$ & $0.055(3)$ & $0.040(2)$ & $0.039(2)$ & $0.017(2)$ & $0.012(2)$ & $0.0233(19)$ \\
\hline OW1 & $0.192(4)$ & $0.069(2)$ & $0.109(3)$ & 0.009 (3) & $0.079(3)$ & $0.028(2)$ \\
\hline OW2 & $0.063(3)$ & $0.086(3)$ & $0.074(3)$ & $0.032(2)$ & $0.020(2)$ & $0.043(2)$ \\
\hline OW3 & $0.192(4)$ & $0.069(2)$ & $0.109(3)$ & $0.009(3)$ & $0.079(3)$ & $0.028(2)$ \\
\hline OW4 & $0.158(6)$ & $0.115(4)$ & $0.103(4)$ & -0.017 (4) & -0.019 & $0.084(4)$ \\
\hline
\end{tabular}


Geometric parameters $\left(\AA,{ }^{\circ}\right)$

\begin{tabular}{|c|c|c|c|}
\hline La1-O5 & $2.507(2)$ & $\mathrm{C} 12-\mathrm{H} 12 \mathrm{~B}$ & 0.9700 \\
\hline $\mathrm{La} 1-\mathrm{O}^{\mathrm{i}}$ & $2.905(3)$ & C14-H14 & 0.9300 \\
\hline $\mathrm{La} 1-\mathrm{O} 3^{\mathrm{ii}}$ & $2.781(2)$ & $\mathrm{C} 14-\mathrm{C} 15^{\mathrm{iii}}$ & $1.399(6)$ \\
\hline $\mathrm{La} 1-\mathrm{O} 3$ & $2.545(2)$ & $\mathrm{C} 8-\mathrm{C} 10$ & $1.373(6)$ \\
\hline $\mathrm{La} 1-\mathrm{O} 1$ & $2.673(2)$ & $\mathrm{C} 8-\mathrm{C} 7$ & $1.509(5)$ \\
\hline La1-O6 & $2.559(2)$ & $\mathrm{C} 8-\mathrm{C} 9$ & $1.388(6)$ \\
\hline $\mathrm{La} 1-\mathrm{O} 2$ & $2.569(2)$ & $\mathrm{C} 10-\mathrm{H} 10$ & 0.9300 \\
\hline $\mathrm{La} 1-\mathrm{O} 4$ & $2.566(2)$ & $\mathrm{C} 10-\mathrm{C}^{\text {iv }}$ & $1.382(6)$ \\
\hline $\mathrm{La} 1-\mathrm{O} 7$ & $2.543(3)$ & $\mathrm{C} 15-\mathrm{H} 15$ & 0.9300 \\
\hline $\mathrm{La} 1-\mathrm{O} 8$ & $2.562(3)$ & $\mathrm{C} 7-\mathrm{H} 7 \mathrm{C}$ & 0.9700 \\
\hline $\mathrm{La} 1-\mathrm{C} 11$ & $3.066(3)$ & C7-H7D & 0.9700 \\
\hline $\mathrm{La} 1-\mathrm{C} 1$ & $2.988(4)$ & C9- $\mathrm{H} 9$ & 0.9300 \\
\hline $\mathrm{O} 5-\mathrm{C}^{\mathrm{i}}$ & $1.255(4)$ & $\mathrm{C} 3-\mathrm{C} 4$ & $1.385(6)$ \\
\hline $\mathrm{O} 3-\mathrm{C} 11^{\mathrm{ii}}$ & $1.264(4)$ & $\mathrm{C} 3-\mathrm{C} 5$ & $1.381(6)$ \\
\hline $\mathrm{O} 1-\mathrm{C} 1$ & $1.264(4)$ & $\mathrm{C} 3-\mathrm{C} 2$ & $1.509(6)$ \\
\hline $\mathrm{O} 6-\mathrm{C} 6$ & $1.256(4)$ & $\mathrm{C} 4-\mathrm{H} 4$ & 0.9300 \\
\hline $\mathrm{O} 2-\mathrm{C} 1$ & $1.248(4)$ & $\mathrm{C} 4-\mathrm{C}^{\mathrm{v}}$ & $1.391(6)$ \\
\hline $\mathrm{O} 4-\mathrm{C} 11$ & $1.246(4)$ & $\mathrm{C} 5-\mathrm{H} 5$ & 0.9300 \\
\hline O7-H7A & 0.824 (19) & $\mathrm{C} 2-\mathrm{H} 2 \mathrm{~A}$ & 0.9700 \\
\hline O7- & $0.806(18)$ & $\mathrm{C} 2-\mathrm{H} 2 \mathrm{~B}$ & 0.9700 \\
\hline $\mathrm{O} 8-\mathrm{H} 8 \mathrm{~A}$ & $0.815(19)$ & OW1-HW1A & $0.87(2)$ \\
\hline O8- $\mathrm{H} 8 \mathrm{~B}$ & $0.833(19)$ & OW1-HW1B & $0.88(2)$ \\
\hline $\mathrm{C} 6-\mathrm{C} 7$ & $1.512(5)$ & OW2-HW2A & $0.821(19)$ \\
\hline $\mathrm{C} 11-\mathrm{C} 12$ & $1.516(5)$ & OW2-HW2B & $0.81(2)$ \\
\hline $\mathrm{C} 1-\mathrm{C} 2$ & $1.514(5)$ & OW3-HW3A & $0.82(2)$ \\
\hline $\mathrm{C} 13-\mathrm{C} 12$ & $1.507(5)$ & OW3-HW3B & $0.81(2)$ \\
\hline $\mathrm{C} 13-\mathrm{C} 14$ & $1.376(6)$ & OW4-HW4A & $0.81(2)$ \\
\hline $\mathrm{C} 13-\mathrm{C} 15$ & $1.371(5)$ & OW4-HW4B & $0.84(2)$ \\
\hline $\mathrm{C} 12-\mathrm{H} 12 \mathrm{~A}$ & 0.9700 & & \\
\hline $\mathrm{O} 5-\mathrm{La} 1-\mathrm{O} 5^{\mathrm{i}}$ & $61.48(9)$ & $\mathrm{C} 11-\mathrm{O} 4-\mathrm{La} 1$ & $101.3(2)$ \\
\hline $\mathrm{O} 5-\mathrm{La} 1-\mathrm{O} 3$ & $146.45(9)$ & $\mathrm{La} 1-\mathrm{O} 7-\mathrm{H} 7 \mathrm{~A}$ & $116(3)$ \\
\hline $\mathrm{O} 5-\mathrm{La} 1-\mathrm{O} 3^{\mathrm{ii}}$ & $118.58(7)$ & $\mathrm{La} 1-\mathrm{O} 7-\mathrm{H} 7 \mathrm{~B}$ & $133(3)$ \\
\hline $\mathrm{O} 5-\mathrm{La} 1-\mathrm{O} 1$ & $75.87(8)$ & $\mathrm{H} 7 \mathrm{~A}-\mathrm{O} 7-\mathrm{H} 7 \mathrm{~B}$ & $109(3)$ \\
\hline $\mathrm{O} 5-\mathrm{La} 1-\mathrm{O} 6$ & $107.86(8)$ & $\mathrm{La} 1-\mathrm{O} 8-\mathrm{H} 8 \mathrm{~A}$ & $118(3)$ \\
\hline $\mathrm{O} 5-\mathrm{La} 1-\mathrm{O} 2$ & $75.29(8)$ & $\mathrm{La} 1-\mathrm{O} 8-\mathrm{H} 8 \mathrm{~B}$ & $122(3)$ \\
\hline $\mathrm{O} 5-\mathrm{La} 1-\mathrm{O} 4$ & $80.33(8)$ & $\mathrm{H} 8 \mathrm{~A}-\mathrm{O} 8-\mathrm{H} 8 \mathrm{~B}$ & $104(3)$ \\
\hline $\mathrm{O} 5-\mathrm{La} 1-\mathrm{O} 7$ & $71.93(9)$ & $\mathrm{O} 5^{\mathrm{i}}-\mathrm{C} 6-\mathrm{La} 1$ & $68.28(18)$ \\
\hline $\mathrm{O} 5-\mathrm{La} 1-\mathrm{O} 8$ & $140.13(9)$ & $\mathrm{O} 5^{\mathrm{i}}-\mathrm{C} 6-\mathrm{O} 6$ & $120.2(3)$ \\
\hline $\mathrm{O} 5-\mathrm{La} 1-\mathrm{C} 11$ & $156.28(8)$ & $\mathrm{O} 5^{\mathrm{i}}-\mathrm{C} 6-\mathrm{C} 7$ & $120.1(3)$ \\
\hline $\mathrm{O} 5-\mathrm{La} 1-\mathrm{C} 11$ & $98.48(8)$ & $\mathrm{O} 6-\mathrm{C} 6-\mathrm{La} 1$ & $52.33(16)$ \\
\hline $\mathrm{O} 5-\mathrm{La} 1-\mathrm{C} 1$ & $75.84(9)$ & $\mathrm{O} 6-\mathrm{C} 6-\mathrm{C} 7$ & $119.7(3)$ \\
\hline $\mathrm{O} 5$ - $\mathrm{La} 1-\mathrm{C} 1$ & $88.69(9)$ & $\mathrm{C} 7-\mathrm{C} 6-\mathrm{La} 1$ & $169.6(2)$ \\
\hline $\mathrm{O} 3-\mathrm{La} 1-\mathrm{O} 5^{\mathrm{i}}$ & $118.22(7)$ & $\mathrm{O}^{\mathrm{ii}}-\mathrm{C} 11-\mathrm{La} 1$ & $65.08(17)$ \\
\hline $\mathrm{O} 3^{\mathrm{ii}}-\mathrm{La} 1-\mathrm{O} 5^{\mathrm{i}}$ & $179.00(7)$ & $\mathrm{O}^{\mathrm{ii}}-\mathrm{C} 11-\mathrm{C} 12$ & $120.3(3)$ \\
\hline $\mathrm{O} 3-\mathrm{La} 1-\mathrm{O} 3^{\mathrm{ii}}$ & $61.11(8)$ & $\mathrm{O} 4-\mathrm{C} 11-\mathrm{La} 1$ & $55.16(17)$ \\
\hline
\end{tabular}




$$
\begin{aligned}
& \text { O3-La1-O1 } \\
& \text { O3-La1-O6 } \\
& \text { O3-La1-O2 } \\
& \text { O3-La1-O4 } \\
& \text { O3-La1-O8 } \\
& \mathrm{O}^{\mathrm{ii}}-\mathrm{La} 1-\mathrm{C} 11 \\
& \text { O3-La1-C11 } \\
& \text { O3-La1-C1 } \\
& \mathrm{O} 3{ }^{\mathrm{ii}}-\mathrm{La} 1-\mathrm{C} 1 \\
& \mathrm{O} 1-\mathrm{La} 1-\mathrm{O} 5^{\mathrm{i}} \\
& \mathrm{O} 1-\mathrm{La} 1-\mathrm{O}^{\mathrm{ii}} \\
& \text { O1-La1-C11 } \\
& \text { O1-La1-C1 } \\
& \text { O6- } \mathrm{La} 1-\mathrm{O} 5^{\mathrm{i}} \\
& \text { O6- } \mathrm{La} 1-\mathrm{O} 3^{\mathrm{ii}} \\
& \text { O6-La1-O1 } \\
& \text { O6-La1-O2 } \\
& \text { O6-La1-O4 } \\
& \text { O6-La1-O8 } \\
& \text { O6-La1-C11 } \\
& \text { O6-La1-C1 } \\
& \mathrm{O} 2-\mathrm{La} 1-\mathrm{O} 5^{\mathrm{i}} \\
& \mathrm{O} 2-\mathrm{La} 1-\mathrm{O}^{3 i} \\
& \text { O2-La1-O1 } \\
& \text { O2-La1-C11 } \\
& \mathrm{O} 2-\mathrm{La} 1-\mathrm{C} 1 \\
& \mathrm{O} 4-\mathrm{La} 1-\mathrm{O}^{\mathrm{i}} \\
& \mathrm{O} 4-\mathrm{La} 1-\mathrm{O} 3^{\mathrm{ii}} \\
& \text { O4-La1-O1 } \\
& \text { O4-La1-O2 } \\
& \text { O4-La1-C11 } \\
& \text { O4-La1-C1 } \\
& \text { O7- } \mathrm{La} 1-\mathrm{O}^{\mathrm{i}} \\
& \text { O7-La1-O3 } \\
& \text { O7- } \mathrm{La} 1-\mathrm{O} 3^{\mathrm{ii}} \\
& \text { O7-La1-O1 } \\
& \text { O7-La1-O6 } \\
& \text { O7-La1-O2 } \\
& \text { O7-La1-O4 } \\
& \text { O7-La1-O8 } \\
& \text { O7-La1-C11 } \\
& \text { O7-La1-C1 } \\
& \mathrm{O} 8-\mathrm{La} 1-\mathrm{O} 5^{\mathrm{i}} \\
& \mathrm{O} 8-\mathrm{La} 1-\mathrm{O} 3^{\mathrm{ii}} \\
& \text { O8-La1-O1 } \\
& \text { O8- } \mathrm{La} 1-\mathrm{O} 2 \\
& \text { O8- } \mathrm{La} 1-\mathrm{O} 4 \\
& \text { O8-La1-C11 }
\end{aligned}
$$

\begin{tabular}{|c|c|c|}
\hline $73.20(8)$ & $\mathrm{O} 4-\mathrm{C} 11-\mathrm{O}^{\mathrm{ii}}$ & $119.9(3)$ \\
\hline $80.93(8)$ & $\mathrm{O} 4-\mathrm{C} 11-\mathrm{C} 12$ & $119.8(3)$ \\
\hline $74.87(8)$ & $\mathrm{C} 12-\mathrm{C} 11-\mathrm{La} 1$ & $171.2(2)$ \\
\hline $108.82(8)$ & $\mathrm{O} 1-\mathrm{C} 1-\mathrm{La} 1$ & $63.39(19)$ \\
\hline $73.39(9)$ & $\mathrm{O} 1-\mathrm{C} 1-\mathrm{C} 2$ & $119.8(3)$ \\
\hline $24.36(8)$ & $\mathrm{O} 2-\mathrm{C} 1-\mathrm{La} 1$ & $58.58(18)$ \\
\hline $85.47(8)$ & $\mathrm{O} 2-\mathrm{C} 1-\mathrm{O} 1$ & $121.5(3)$ \\
\hline $70.64(9)$ & $\mathrm{O} 2-\mathrm{C} 1-\mathrm{C} 2$ & $118.7(3)$ \\
\hline $90.37(9)$ & $\mathrm{C} 2-\mathrm{C} 1-\mathrm{La} 1$ & $172.4(3)$ \\
\hline $109.68(7)$ & $\mathrm{C} 14-\mathrm{C} 13-\mathrm{C} 12$ & $120.5(4)$ \\
\hline $69.49(8)$ & $\mathrm{C} 15-\mathrm{C} 13-\mathrm{C} 12$ & $122.1(4)$ \\
\hline $74.29(9)$ & $\mathrm{C} 15-\mathrm{C} 13-\mathrm{C} 14$ & $117.4(4)$ \\
\hline $25.01(9)$ & $\mathrm{C} 11-\mathrm{C} 12-\mathrm{H} 12 \mathrm{~A}$ & 109.2 \\
\hline $46.41(7)$ & $\mathrm{C} 11-\mathrm{C} 12-\mathrm{H} 12 \mathrm{~B}$ & 109.2 \\
\hline $133.56(8)$ & $\mathrm{C} 13-\mathrm{C} 12-\mathrm{C} 11$ & $112.0(3)$ \\
\hline $126.21(9)$ & $\mathrm{C} 13-\mathrm{C} 12-\mathrm{H} 12 \mathrm{~A}$ & 109.2 \\
\hline $78.86(9)$ & $\mathrm{C} 13-\mathrm{C} 12-\mathrm{H} 12 \mathrm{~B}$ & 109.2 \\
\hline $149.49(10)$ & $\mathrm{H} 12 \mathrm{~A}-\mathrm{C} 12-\mathrm{H} 12 \mathrm{~B}$ & 107.9 \\
\hline $71.55(10)$ & $\mathrm{C} 13-\mathrm{C} 14-\mathrm{H} 14$ & 119.1 \\
\hline $149.62(9)$ & $\mathrm{C} 13-\mathrm{C} 14-\mathrm{C} 15^{\mathrm{iii}}$ & $121.8(4)$ \\
\hline $101.90(10)$ & 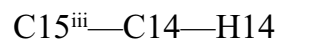 & 119.1 \\
\hline $66.57(8)$ & $\mathrm{C} 10-\mathrm{C} 8-\mathrm{C} 7$ & $121.6(4)$ \\
\hline $112.44(8)$ & $\mathrm{C} 10-\mathrm{C} 8-\mathrm{C} 9$ & $117.8(4)$ \\
\hline $49.39(8)$ & $\mathrm{C} 9-\mathrm{C} 8-\mathrm{C} 7$ & $120.6(4)$ \\
\hline $123.42(9)$ & $\mathrm{C} 8-\mathrm{C} 10-\mathrm{H} 10$ & 119.5 \\
\hline $24.50(9)$ & $\mathrm{C} 8-\mathrm{C} 10-\mathrm{C}^{\mathrm{iv}}$ & $121.1(4)$ \\
\hline $132.95(7)$ & $\mathrm{C} 9^{\mathrm{iv}}-\mathrm{C} 10-\mathrm{H} 10$ & 119.5 \\
\hline $47.75(7)$ & $\mathrm{C} 13-\mathrm{C} 15-\mathrm{C} 14^{\mathrm{iii}}$ & $120.7(4)$ \\
\hline $84.13(9)$ & $\mathrm{C} 13-\mathrm{C} 15-\mathrm{H} 15$ & 119.6 \\
\hline $131.27(9)$ & 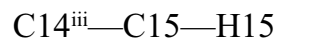 & 119.6 \\
\hline $23.49(8)$ & $\mathrm{C} 6-\mathrm{C} 7-\mathrm{H} 7 \mathrm{C}$ & 108.9 \\
\hline $108.61(10)$ & $\mathrm{C} 6-\mathrm{C} 7-\mathrm{H} 7 \mathrm{D}$ & 108.9 \\
\hline $70.81(9)$ & $\mathrm{C} 8-\mathrm{C} 7-\mathrm{C} 6$ & $113.4(3)$ \\
\hline $141.53(10)$ & $\mathrm{C} 8-\mathrm{C} 7-\mathrm{H} 7 \mathrm{C}$ & 108.9 \\
\hline $110.19(9)$ & $\mathrm{C} 8-\mathrm{C} 7-\mathrm{H} 7 \mathrm{D}$ & 108.9 \\
\hline $142.43(10)$ & $\mathrm{H} 7 \mathrm{C}-\mathrm{C} 7-\mathrm{H} 7 \mathrm{D}$ & 107.7 \\
\hline $82.59(10)$ & $\mathrm{C} 8-\mathrm{C} 9-\mathrm{H} 9$ & 119.4 \\
\hline $134.87(9)$ & $\mathrm{C} 10^{\mathrm{iv}}-\mathrm{C} 9-\mathrm{C} 8$ & $121.1(4)$ \\
\hline $71.95(10)$ & $\mathrm{C} 10^{\mathrm{iv}}-\mathrm{C} 9-\mathrm{H} 9$ & 119.4 \\
\hline $68.46(10)$ & $\mathrm{C} 4-\mathrm{C} 3-\mathrm{C} 2$ & $120.7(4)$ \\
\hline $91.69(10)$ & $\mathrm{C} 5-\mathrm{C} 3-\mathrm{C} 4$ & $118.0(4)$ \\
\hline $147.21(10)$ & $\mathrm{C} 5-\mathrm{C} 3-\mathrm{C} 2$ & $121.2(4)$ \\
\hline $108.04(8)$ & $\mathrm{C} 3-\mathrm{C} 4-\mathrm{H} 4$ & 119.8 \\
\hline $72.59(9)$ & $\mathrm{C} 3-\mathrm{C} 4-\mathrm{C} 5^{\mathrm{v}}$ & $120.4(4)$ \\
\hline $138.11(8)$ & $\mathrm{C} 5^{v}-\mathrm{C} 4-\mathrm{H} 4$ & 119.8 \\
\hline $139.28(10)$ & $\mathrm{C} 3-\mathrm{C} 5-\mathrm{C} 4^{\mathrm{v}}$ & $121.6(4)$ \\
\hline $83.41(11)$ & $\mathrm{C} 3-\mathrm{C} 5-\mathrm{H} 5$ & 119.2 \\
\hline $78.57(10)$ & $\mathrm{C} 44^{\mathrm{v}}-\mathrm{C} 5-\mathrm{H} 5$ & 119.2 \\
\hline
\end{tabular}




$\begin{array}{llll}\text { O8-La1-C1 } & 144.03(10) & \mathrm{C} 1-\mathrm{C} 2-\mathrm{H} 2 \mathrm{~A} & 109.0 \\ \mathrm{C} 1-\mathrm{La} 1-\mathrm{C} 11 & 98.95(10) & \mathrm{C} 1-\mathrm{C} 2-\mathrm{H} 2 \mathrm{~B} & 109.0 \\ \mathrm{~L} a 1-\mathrm{O} 5-\mathrm{La} 1^{\mathrm{i}} & 118.52(9) & \mathrm{C} 3-\mathrm{C} 2-\mathrm{C} 1 & 112.8(3) \\ \mathrm{C} 6 \text { - }-\mathrm{O} 5-\mathrm{La}^{\mathrm{i}} & 88.1(2) & \mathrm{C} 3-\mathrm{C} 2-\mathrm{H} 2 \mathrm{~A} & 109.0 \\ \mathrm{C} 6-\mathrm{O} 5-\mathrm{L} 1 & 153.4(2) & \mathrm{C} 3-\mathrm{C} 2-\mathrm{H} 2 \mathrm{~B} & 109.0 \\ \mathrm{~L} 11-\mathrm{O} 3-\mathrm{La} 1^{\mathrm{ii}} & 118.89(8) & \mathrm{H} 2 \mathrm{~A}-\mathrm{C} 2-\mathrm{H} 2 \mathrm{~B} & 107.8 \\ \mathrm{C} 11^{\mathrm{ii}}-\mathrm{O} 3-\mathrm{La} 1 & 150.5(2) & \mathrm{HW} 1 \mathrm{~A}-\mathrm{OW} 1-\mathrm{HW} 1 \mathrm{~B} & 88(7) \\ \mathrm{C} 11^{\mathrm{ii}}-\mathrm{O} 3-\mathrm{La} 1^{\mathrm{ii}} & 90.56(19) & \mathrm{HW} 2 \mathrm{~A}-\mathrm{OW} 2-\mathrm{HW} 2 \mathrm{~B} & 121(5) \\ \mathrm{C} 1-\mathrm{O} 1-\mathrm{La} 1 & 91.6(2) & \mathrm{HW} 3 \mathrm{~A}-\mathrm{OW}-\mathrm{HW} 3 \mathrm{~B} & 108(4) \\ \mathrm{C} 6-\mathrm{O} 6-\mathrm{La} 1 & 104.8(2) & \text { HW4A-OW4-HW4B } & 107(4) \\ \mathrm{C} 1-\mathrm{O} 2-\mathrm{La} 1 & 96.9(2) & & \end{array}$

Symmetry codes: (i) $-x+1,-y+1,-z+1$; (ii) $-x+2,-y+1,-z+1$; (iii) $-x+1,-y,-z$; (iv) $-x+2,-y+2,-z+2$; (v) $-x+2,-y+1,-z+2$.

Hydrogen-bond geometry $\left(A,{ }^{\circ}\right)$

\begin{tabular}{|c|c|c|c|c|}
\hline$D-\mathrm{H} \cdots A$ & $D-\mathrm{H}$ & $\mathrm{H} \cdots A$ & $D \cdots A$ & $D-\mathrm{H} \cdots A$ \\
\hline $\mathrm{O} W 1-\mathrm{H} W 1 A \cdots \mathrm{O} W 1^{\mathrm{vi}}$ & $0.87(9)$ & $2.40(11)$ & $3.067(13)$ & $133(9)$ \\
\hline $\mathrm{O} W 1-\mathrm{H} W 1 B \cdots \mathrm{O} W 4^{\mathrm{vii}}$ & $0.89(9)$ & $2.54(10)$ & $3.298(11)$ & $145(7)$ \\
\hline $\mathrm{O} W 2-\mathrm{H} W 2 A \cdots \mathrm{O} 4^{\mathrm{i}}$ & $0.82(6)$ & $2.10(6)$ & $2.895(5)$ & $164(6)$ \\
\hline $\mathrm{O} W 2-\mathrm{H} W 2 B \cdots \mathrm{O} W 4^{\text {viii }}$ & $0.82(6)$ & $2.20(5)$ & $2.855(8)$ & $137(5)$ \\
\hline $\mathrm{O} W 3-\mathrm{H} W 3 A \cdots \mathrm{O}^{\mathrm{i}}$ & $0.82(8)$ & $2.02(8)$ & $2.780(8)$ & $154(7)$ \\
\hline $\mathrm{O} W 3-\mathrm{H} W 3 B \cdots \mathrm{O} W 1^{\mathrm{vi}}$ & $0.81(7)$ & $2.40(8)$ & $3.162(11)$ & $156(8)$ \\
\hline $\mathrm{O} W 4-\mathrm{H} W 4 A \cdots \mathrm{O} W 2^{\mathrm{ix}}$ & $0.81(10)$ & $2.49(9)$ & $2.855(8)$ & $109(9)$ \\
\hline $\mathrm{O} 7-\mathrm{H} 7 A \cdots \mathrm{O} 2^{\mathrm{i}}$ & $0.82(4)$ & $1.95(4)$ & $2.741(5)$ & $161(5)$ \\
\hline $\mathrm{O} 7-\mathrm{H} 7 B \cdots \mathrm{O} W 4$ & $0.81(5)$ & $2.03(5)$ & $2.800(9)$ & $160(5)$ \\
\hline $\mathrm{O} W 4-\mathrm{H} W 4 B \cdots \mathrm{O} W 3^{\mathrm{x}}$ & $0.84(9)$ & $2.11(10)$ & $2.824(11)$ & $143(8)$ \\
\hline $\mathrm{O} 8-\mathrm{H} 8 A \cdots \mathrm{O} W 3^{\mathrm{i}}$ & $0.82(4)$ & $2.38(4)$ & $3.175(8)$ & $165(4)$ \\
\hline $\mathrm{O} 8-\mathrm{H} 8 B^{\cdots} \cdots \mathrm{O} 1^{\mathrm{ii}}$ & $0.83(4)$ & $1.92(4)$ & $2.725(5)$ & $163(5)$ \\
\hline $\mathrm{C} 7-\mathrm{H} 7 D^{\cdots} \cdots 4^{\mathrm{i}}$ & 0.97 & 2.54 & $3.442(6)$ & 154 \\
\hline $\mathrm{C} 12-\mathrm{H} 12 B \cdots \mathrm{O}^{6 i}$ & 0.97 & 2.51 & $3.406(6)$ & 154 \\
\hline
\end{tabular}

Symmetry codes: (i) $-x+1,-y+1,-z+1$; (ii) $-x+2,-y+1,-z+1$; (vi) $-x+1,-y,-z+1$; (vii) $x, y-1, z$; (viii) $x, y, z+1$; (ix) $x, y, z-1$; (x) $x+1, y+1, z$. 